\title{
*-Autonomous categories and linear logic
}

\author{
Michael Barr* \\ Department of Mathematics and Statistics \\ McGill University \\ Montreal, Quebec, Canada
}

1999-06-11

\section{Introduction}

The subject of linear logic has recently become very important in theoretical computer science. It is apparent that the *-autonomous categories studied at length in [Barr, 1979] are a model for a large fragment of linear logic, although not quite for the whole thing. Since the main reference is out of print and since large parts of that volume are devoted to results highly peripheral to the matter at hand, it seemed reasonable to provide a short introduction to the subject.

I include here the formal construction of my student Po-Hsiang Chu that appears as an appendix to that volume. At the time, the formal construction appeared not to have substantial mathematical interest, but it appears to be the most interesting part in the present context. It turns out, for example, that one can in many cases construct models of the full linear logic from Chu's construction applied to a cartesian closed category.

One problem in this subject is that having two very different sources - from linear logic as well as category theory - two very different and essentially inconsistent notations have arisen. Without much hope, I will attempt here to introduce a terminology and notation that draws from both subjects and is as consistent as possible with the two different traditions. To begin with, there is the name *-autonomous category that gives the $*$ notation a privileged position. (It also gives librarians fits, which is a good reason to keep using it.) The notation using $\perp$ is certainly suggestive, but the name "perp-autonomous" categories is graceless. Thus I will continue to call them $*$-autonomous categories for the time being. The notations $0,1,+, \times$ for categorical initial object, final object, sum and product are too hallowed by use to even think of changing. Everyone agrees on $\otimes$ for the tensor. Then there is the dual construction,

\footnotetext{
* In the preparation of this paper, I have been assisted by a grant from the NSERC of Canada. I would also like to thank McGill University for a sabbatical leave and the University of Pennsylvania for a very congenial setting in which to spend that leave.
} 
for which an upside-down ampersand has been used. Aside from the fact that it is hard to make without considerable practice, it cannot be done on $\mathrm{TEX}_{\mathrm{E}}$ or most other typesetting systems. Although various alternates have been tried, it seems to me that the $*$ is the best for this purpose; it is easy to make and to print and, also important, it is not likely to get confused with the $\otimes$, even when carelessly drawn. As for what to call it, let us just call it dual tensor until something better comes along ("cotensor" would be good, but it has another meaning). The unit for the tensor is usually called $I$, but there is nothing really sacred about that and $T$ seems like a reasonable choice. The dual of $T$ is obviously $\perp$ and I will use this. This has the unfortunate side-effect that what we call $A^{\perp}$ is what in another notation is called $\perp^{A}$. As far as I am concerned, this is no loss; that notation is outdated and ought to be abandoned anyway. What I will use in its stead is Girard's quite attractive $-\circ$. So the basic adjunction isomorphism is

$$
\operatorname{Hom}(A \otimes B, C) \cong \operatorname{Hom}(B, A \multimap C)
$$

\section{$2 *$-Autonomous categories}

By an autonomous category I mean a closed symmetric monoidal category. It will be called *-autonomous category if it has, in addition, an object denoted $\perp$, called the dualizing object, such that for any object $A$, the canonical map to the second dual of $A$ with respect to $\perp$ is an isomorphism.

More precisely, let $\mathscr{V}$ be a category. Then $\mathscr{V}$ is monoidal if there is a bifunctor $-\otimes-: \mathscr{V} \times \mathscr{V} \longrightarrow \mathscr{V}$, an object $\top$ and isomorphisms

$$
\begin{gathered}
a(A, B, C):(A \otimes B) \otimes C \longrightarrow A \otimes(B \otimes C) \\
r(A): A \otimes \top \longrightarrow A \quad \text { and } \quad l(A): \top \otimes A \longrightarrow A
\end{gathered}
$$

defined and natural for all objects $A, B$ and $C$ of $\mathscr{A}$. These are subject to coherence conditions that essentially mean that no non-trivial automorphisms can be constructed using only these arrows. See [Eilenberg \& Kelly] for more details on this and later coherences.

The monoidal category is symmetric if there are isomorphisms

$$
s(A, B): A \otimes B \longrightarrow B \otimes A
$$

that are subject to similar naturality and coherence conditions. However, in this case, it is not true that the coherence prevents there being non-trivial automorphisms since the isomorphism $s(A, A): A \otimes A \longrightarrow A \otimes A$ is generally non-trivial. The monoidal category is closed if there is a bifunctor $-\multimap-: \mathscr{V}^{\mathrm{op}} \times \mathscr{V} \longrightarrow \mathscr{V}$ such that for each object $A$ of $\mathscr{V}$, the functor $A \multimap-$ is left adjoint to $A \otimes-$. This means that for any objects $B$ and $C$ of $\mathscr{V}$, we have

$$
\operatorname{Hom}(A \otimes B, C) \cong \operatorname{Hom}(B, A \multimap C)
$$


and these isomorphisms are natural in $B$ and $C$.

No special coherence has to be supposed for $-\multimap-$ as it will follow from the coherence of the $\otimes$ and adjointness. It is purely formal, for example, that $\top \multimap A \cong A$ and that

$$
(A \otimes B) \multimap C \cong B \multimap(A \multimap C)
$$

Given any object $\perp$ in an autonomous category, we have, for each object $A$, the identity arrow $A \multimap \perp \longrightarrow A \multimap \perp$. This corresponds under the adjointness to an arrow $A \otimes(A \multimap \perp) \longrightarrow \perp$ which is called evaluation. By composing with the symmetry $s(A \multimap \perp, A)$, we get an arrow $(A \multimap \perp) \otimes A \longrightarrow \perp$ and this corresponds to an arrow $A$ $\longrightarrow(A \multimap \perp) \multimap \perp . \perp$ is called a dualizing object if this latter object is an isomorphism for every object $A$ of $\mathscr{V}$. An autonomous category with a dualizing object is a *-autonomous category. It will be convenient to write $A^{\perp}$ for $A \multimap \perp$.

In many, but not all cases, the dualizing object will be the object $T$. However there is nothing in the definition that requires it. In [Barr, 1979] Chapter IV, Section 2 an example is given in which it is not that object. In fact, the category includes the locally compact abelian groups and the dualizing object is the circle group. Also, the dualizing object is not necessarily unique (same reference as above). Nonetheless, we will abuse notation and suppress mention of the dualizing object for the most part.

Some of the useful properties of $*$-autonomous categories include

1. $\operatorname{Hom}(A, B) \cong \operatorname{Hom}\left(B^{\perp}, A^{\perp}\right)$; in fact, $A \multimap B \cong B^{\perp} \multimap A^{\perp}$.

2. $A \otimes B \cong\left(A \multimap B^{\perp}\right)^{\perp}$; equivalently $A \multimap B \cong\left(A \otimes B^{\perp}\right)^{\perp}$.

One thing that is not generally true (and the $*$-autonomous is called compact if it is) are either of the two equivalent:

1. $A \multimap B \cong A^{\perp} \otimes B$.

2. $(A \otimes B)^{\perp} \cong A^{\perp} \otimes B^{\perp}$.

Here are a couple of interesting examples. The first - and probably prototypical - is the category of finite dimensional vector spaces over a field. The (unique) dualizing object is the field itself. The details are familiar. Notable is the fact that this duality does not extend to infinite dimensional spaces. In fact $V \cong V^{\perp \perp}$ is equivalent to $V$ being finite dimensional. The finite dimensional vector spaces are a compact category.

Another example is the category of complete inf semilattices. This is a poset in which every set of elements has a greatest lower bound (inf). It is evident that every set of elements also has a least upper bound (sup), but the arrows in this category preserve only the infs.

The set of such functions between two such lattices is again a complete inf lattice with pointwise inf. It is easy to see that the 2 element lattice is a dualizing object. In 
fact, if $L$ is such a lattice, every map $f: L \longrightarrow 2$ has the form

$$
f(x)= \begin{cases}1 & \text { if } x \geq x_{0} \\ 0 & \text { otherwise }\end{cases}
$$

for a unique element $x_{0} \in L$ (namely the inf of all $x$ such that $f(x)=1$ ). From this it is easy to see that the dual of $L$ is simply $L^{\text {op }}$, the same elements but with the order reversed. If $f: L \longrightarrow L^{\prime}$ is an arrow in the category, the arrow $f^{\perp}: L^{\prime \text { op }} \longrightarrow L^{\text {op }}$ can be simply described as the left adjoint of $f$, when $L$ and $L^{\prime}$ are considered as categories. This example is not compact (even for finite semilattices).

The category of sets and relations (an arrow from $X \longrightarrow Y$ is a subset of $X \times Y$ ) is a $*$-autonomous, in fact compact, category. Both $X \otimes Y$ and $X \multimap Y$ are just the ordinary cartesian product $X \times Y$ (which is not the cartesian product in the category). The adjunction is immediate; both $\operatorname{Hom}(X \times Y, Z)$ and $\operatorname{Hom}(X, Y \multimap Z)$ (along with a number of other permutations of these symbols) is the set of subsets of $X \times Y \times Z$. One easily sees that the one element set is a dualizing object and that $X^{\perp}=X$ for all objects $X$.

A diagram in a *-autonomous category has a limit if and only if the dual diagram has a colimit. It follows that the category is complete if and only if it is cocomplete. The category of finite dimensional vector spaces has finite limits and colimits, but not very many infinite ones. The category of complete inf semi-lattices is evidently complete and therefore cocomplete. The category of sets and relations has all sums (the usual disjoint unions) and therefore all products. It does not have all equalizers, but it does have the equalizer of a set of automorphisms, a fact we will require later.

\section{Chu's construction}

In [Barr, 1979], most of the text is devoted to constructing complete $*$-autonomous categories beginning from well-known dualities. The paper of P.-H. Chu [1979] is an appendix to that volume and gives a formal construction that is probably of more interest in giving models of linear logic. Thus we give that construction in some detail.

One important point has to be made. Chu referred to a forthcoming coherence result by Szabo for a number of his proofs. This paper has never appeared and Szabo's claims have subsequently been called into question. It is not my purpose to enter into this debate. The coherence results that $\mathrm{Chu}$ needs can be gotten in one of two ways. First off, one can suppose that the category that Chu begins with has a faithful unit object. This means that the unit object $T$ for the tensor product is a generator. In many of the examples as above, this hypothesis is correct. The second possibility is to use the metatheorem of [Jay, 1989] (see especially Section 5). Both of these allow one to derive the required coherence results by using "elements" and that makes it easy. In all cases, the only question at hand is whether a certain diagram, constructed using only the arrows from the free autonomous category, commutes. 
This construction was motivated, by the way, by certain formal constructions in the theory of topological vector spaces, in which one considers a pair consisting of a space and a space of functionals on the space.

All the material of this section has been taken from Chu's paper. For the most part, the construction is given, but the detailed proofs are omitted.

3.1 The setting. We begin with a finitely complete autonomous category $\mathscr{V}$ and a fixed object of $\mathscr{V}$ we denote $\perp$. We will construct a $*$-autonomous "completion" $\mathscr{V}_{\perp}$ in which $\perp$ becomes the dualizing object. In the next section we will show that if $\mathscr{V}$ is complete and cocomplete, so is $\mathscr{V}_{\perp}$.

For any object $V$ of $\mathscr{V}$ we denote $V \multimap \perp$ by $V^{\perp}$ and if $f: V \rightarrow W$, the induced arrow by $f^{\perp}: W^{\perp} \longrightarrow V^{\perp}$.

3.2 The construction. An object of $\mathscr{V}_{\perp}$ is a triplet $\left(V, V^{\prime}, v\right)$ where $V$ and $V^{\prime}$ are objects of $\mathscr{V}$ and $v: V \otimes V^{\prime} \longrightarrow \perp$ is an arrow of $\mathscr{V}$. A morphism is a pair $\left(f, f^{\prime}\right):\left(V, V^{\prime}, v\right) \longrightarrow\left(W, W^{\prime}, w\right)$ where $f: V \longrightarrow W$ and $f^{\prime}: W^{\prime} \longrightarrow V^{\prime}$ (note the direction!) such that the diagram

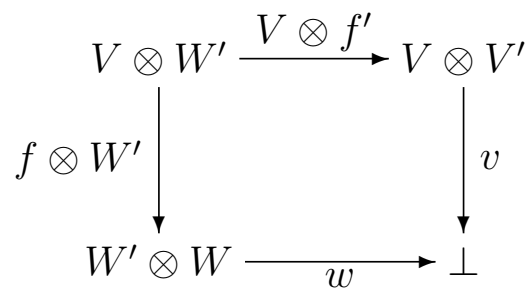

commutes. The arrow $v: V \otimes V^{\prime} \longrightarrow \perp$ has transposes $V^{\prime} \longrightarrow V^{\perp}$ and $V \longrightarrow V^{\perp}$ which we will use without further comment.

The following is an immediate consequence of the definition.

3.3 Proposition. Let $\left(V, V^{\prime}, v\right)$ and $\left(W, W^{\prime}, w\right)$ be two objects of $\mathscr{V}_{\perp}$ and let $f: V$ $\longrightarrow W$ and $f^{\prime}: W^{\prime} \longrightarrow V^{\prime}$ be arrows of $\mathscr{V}$. Then a necessary and sufficient condition that $\left(f, f^{\prime}\right)$ be an arrow from $\left(V, V^{\prime}, v\right)$ to $\left(W, W^{\prime}, w\right)$ is that either (and hence both) of the two diagrams commute:
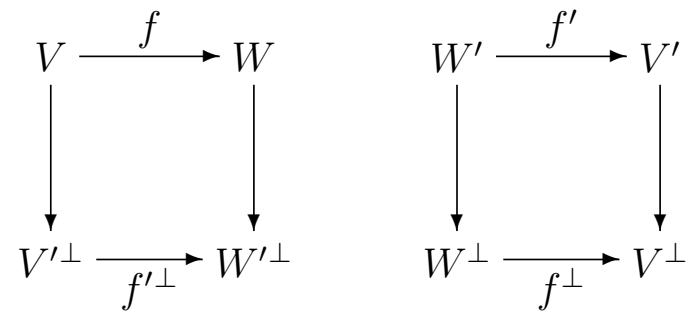

An immediate consequence of this is that if $W \longrightarrow W^{\prime \perp}$ is monic, then $f$ is determined uniquely by $f^{\prime}$ and that if $V^{\prime} \longrightarrow V^{\perp}$ is monic, then $f^{\prime}$ is determined uniquely by $f$. 
In order to define the closed structure on $\mathscr{V}_{\perp}$, we first define a bifunctor

$$
\mathscr{V}(-,-): \mathscr{V}_{\perp}^{\mathrm{op}} \times \mathscr{V}_{\perp} \longrightarrow \mathscr{V}
$$

as the internalization of the above homsets. For objects $A=\left(V, V^{\prime}, v\right)$ and $B=$ $\left(W, W^{\prime}, w\right)$, the following is to be a pullback:

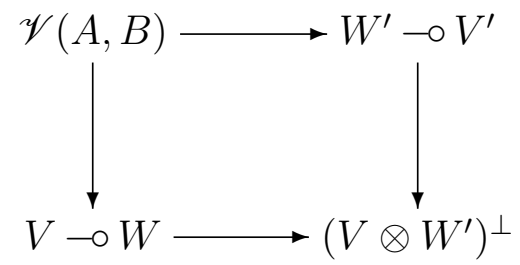

The right hand arrow, for example, is the transpose of the arrow

$$
V \otimes W^{\prime} \otimes\left(W^{\prime} \multimap V^{\prime}\right) \stackrel{V \otimes \text { eval }}{\longrightarrow} V \otimes V^{\prime} \stackrel{v}{\longrightarrow} \perp
$$

and the lower arrow is defined similarly. Then we define

$$
A \multimap B=\left(\mathscr{V}(A, B), V \otimes W^{\prime}, u\right)
$$

where $u$ is defined to be either of the two equal composites

$\mathscr{V}(A, B) \otimes V \otimes W^{\prime} \longrightarrow(V \multimap W) \otimes V \otimes W^{\prime} \longrightarrow V \otimes(V \longrightarrow W) \otimes W^{\prime} \longrightarrow W \otimes W^{\prime} \longrightarrow \perp$

$\mathscr{V}(A, B) \otimes V \otimes W^{\prime} \longrightarrow\left(W^{\prime} \multimap V^{\prime}\right) \otimes V \otimes W^{\prime} \longrightarrow V \otimes W^{\prime} \otimes\left(W^{\prime} \multimap V^{\prime}\right) \longrightarrow V \otimes V^{\prime} \longrightarrow \perp$

We also define, for an object $A=\left(V, V^{\prime}, v\right)$, the dual $A^{\perp}=\left(V^{\prime}, V, v \circ s\right)$, where $s$ is the symmetry of the tensor. One can easily show that the object $T=(\top, \perp, r$ ) (recall that $r: \top \otimes \perp \longrightarrow \perp$ is the right unit isomorphism) has the property that $\top \multimap A \cong A$ for each object $A$ of $\mathscr{V}_{\perp}$. Then it is formal to show that if we define $A \otimes B=\left(A-\circ B^{\perp}\right)^{\perp}$, we have a $*$-autonomous category whose dualizing object is $\top^{\perp}=(\perp, \top, l)$.

\section{Completeness properties}

In this section, we want to show that if $\mathscr{V}$ is complete and cocomplete then so is $\mathscr{V}_{\perp}$. Not surprisingly, given that $\mathscr{V}_{\perp}$ is self dual and consequently complete if and only if cocomplete, neither completeness nor cocompleteness by itself will have such an implication for $\mathscr{V}_{\perp}$. In the remainder of this section, we will assume $\perp$ fixed and write $\mathscr{A}$ for $\mathscr{V}_{\perp}$.

Let $\left\{\left(V_{i}, V_{i}^{\prime}, v_{i}\right)\right\}, i \in I$ be a family of objects of $\mathscr{V}_{\perp}$. Let $V=\sum V_{i}$ and $V^{\prime}=\prod V_{i}^{\prime}$. Since $\otimes$ has a right adjoint, it commutes with sums. Thus there is a unique arrow

$$
v: V \otimes V^{\prime}=\sum_{i}\left(V_{i} \otimes V^{\prime}\right) \longrightarrow \perp
$$


such that $v \mid V_{i} \otimes V^{\prime}$ is the composite

$$
V_{i} \otimes V^{\prime} \stackrel{V_{i} \otimes p_{i}}{\longrightarrow} V_{i} \otimes V_{i}^{\prime} \stackrel{v_{i}}{\longrightarrow} \perp
$$

where $p_{i}: V^{\prime} \longrightarrow V_{i}^{\prime}$ is the projection on the $i$ th coordinate.

4.1 Proposition. In the category $\mathscr{A},\left(V, V^{\prime}, v\right)$ is the sum of the family

$$
\left\{\left(V_{i}, V_{i}^{\prime}, v_{i}\right)\right\}
$$

Proof. What must be shown is that if $c_{i}$ : $V_{i} \longrightarrow V$ is the inclusion of the $i$ th summand, then $\left(c_{i}, p_{i}\right):\left(V_{i}, V_{i}^{\prime}, v_{i}\right) \longrightarrow\left(V, V^{\prime}, v\right)$ is a family of arrows of $\mathscr{A}$ and is the universal such arrow. Being an arrow requires that the diagram

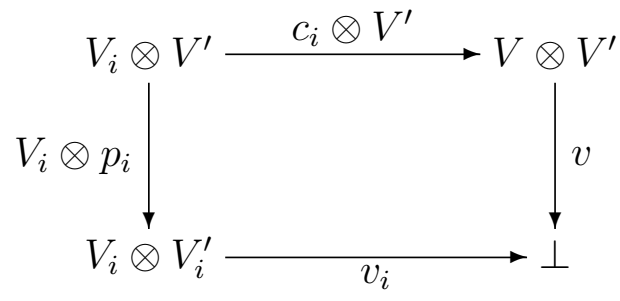

commute. But the definition of $v$ is exactly that commutation. If we have a family $\left\{\left(f_{i}, f_{i}^{\prime}\right):\left(V_{i}, V_{i}^{\prime}, v_{i}\right) \longrightarrow\left(W, W^{\prime}, w\right)\right\}$, then the universal mapping properties of sum and product guarantee the existence of unique arrows $f: V \longrightarrow W$ and $f^{\prime}: W^{\prime} \longrightarrow V^{\prime}$ such that $f \circ c_{i}=f_{i}$ and $p_{i} \circ f^{\prime}=f_{i}^{\prime}$. The only thing to check is that $\left(f, f^{\prime}\right):\left(V, V^{\prime}, v\right)$ $\longrightarrow\left(W, W^{\prime}, w\right)$ is an arrow in $\mathscr{A}$, which I leave to the reader.

Suppose that $\left(f, f^{\prime}\right)$ and $\left(g, g^{\prime}\right)$ are each arrows from $\left(V, V^{\prime}, v\right)$ to $\left(W, W^{\prime}, w\right)$. Let $h: W \longrightarrow U$ be the coequalizer of $f$ and $f^{\prime}$ and $h^{\prime}: U^{\prime} \longrightarrow W^{\prime}$ be the equalizer of $g$ and $g^{\prime}$. In the diagram

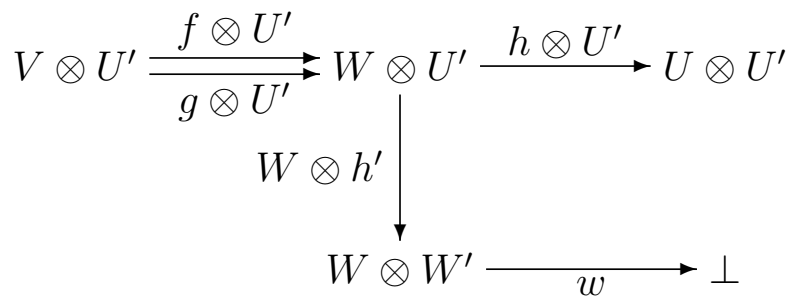

we have that

$$
\begin{aligned}
w \circ W \otimes h^{\prime} \circ f \otimes U^{\prime} & =w \circ f \otimes h^{\prime}=w \circ f \otimes W^{\prime} \circ V \otimes h^{\prime}=v \circ V \otimes f^{\prime} \circ V \otimes h^{\prime} \\
& =v \circ V \otimes\left(f^{\prime} \circ h^{\prime}\right)=v \circ V \otimes\left(g^{\prime} \circ h^{\prime}\right)
\end{aligned}
$$

which by the same argument reduces to $w \circ W \otimes h^{\prime} \circ g \otimes U^{\prime}$. Since $\otimes$ has a right adjoint, it preserves colimits so that the upper row of that diagram is a coequalizer and there results a unique arrow $u: U \otimes U^{\prime} \longrightarrow \perp$ so that $u \circ h \otimes U^{\prime}=w \circ W \otimes h$ which defines an object $\left(U, U^{\prime}, u\right)$ of $\mathscr{A}$ and also shows that $\left(h, h^{\prime}\right):\left(W, W^{\prime}, w\right) \longrightarrow\left(U, U^{\prime}, u\right)$ is an arrow of $\mathscr{A}$. 
4.2 Proposition. In $\mathscr{A}$, the object $\left(U, U^{\prime}, u\right)$ and the arrow $\left(h . h^{\prime}\right):\left(W, W^{\prime}, w\right)$ $\longrightarrow\left(U, U^{\prime}, u\right)$ are a coequalizer of $\left(f, f^{\prime}\right)$ and $\left(g, g^{\prime}\right)$.

Proof. Most the work is already done. If $\left(k, k^{\prime}\right):\left(W, W^{\prime}, w\right) \longrightarrow\left(T, T^{\prime}, t\right)$ is a pair of arrows that coequalize $\left(f, f^{\prime}\right)$ and $\left(g, g^{\prime}\right)$, then we have that $k \circ f=k \circ g$ so that there is a unique arrow $l: U \longrightarrow T$ such that $l \circ h=k$. Similarly, from $f^{\prime} \circ k^{\prime}=g^{\prime} \circ k^{\prime}$, there is a unique arrow $l^{\prime}: T^{\prime} \longrightarrow U^{\prime}$ such that $h^{\prime} \circ l^{\prime}=k^{\prime}$. It remains only to show that $\left(l, l^{\prime}\right):\left(U, U^{\prime}, u\right) \longrightarrow\left(T, T^{\prime}, t\right)$ is an arrow of $\mathscr{A}$, which we leave to the reader.

We have now proved,

4.3 Theorem. Let $\mathscr{V}$ be a complete and cocomplete autonomous category. Then for any object $\perp$ of $\mathscr{V}$, the category $\mathscr{V}_{\perp}$ is a complete and cocomplete $*$-autonomous category.

\section{$5 \quad *$-Autonomous categories and linear logic}

A model of full linear logic is a $*$-autonomous category equipped with one more structure: a cotriple $\mathscr{G}=(!, \epsilon, \delta)$ which has the additional property that $!(A \times B) \cong ! A \otimes ! B$. The likeliest place for such a functor to come from is from a cofree counitary coassociative cocommutative coalgebra. Let us see why this is so. It will be convenient to dualize this discussion, since algebras are so much more familiar than coalgebras.

Let $\mathscr{C}$ be a category with a coherently associative biproduct we will denote $*$ and unit $\top$. Let $\mathscr{B}$ denote the category of unitary associative commutative algebras. There is an underlying functor $U: \mathscr{B} \longrightarrow \mathscr{C}$. The functor $U$ creates limits which means, essentially, that to find the limit of a diagram of algebras, take the limit in $\mathscr{C}$ of the underlying objects, if it exists. that object will have a unique structure map that makes it into the limit in $\mathscr{B}$. As for colimits, the situation is a bit different. In general, very little can be said. In the case of unitary associative commutative algebras, the situation is different. In fact, if $B_{1}$ and $B_{2}$ are algebras, the coproduct is given by the object $U B_{1} * U B_{2}$ with an appropriate structure. Since free functors preserve colimits, it follows that

$$
T\left(C_{1}+C_{2}\right)=U F\left(C_{1}+C_{2}\right) \cong U\left(F C_{1}+F C_{2}\right) \cong U F C_{1} * U F C_{2}=T C_{1} * T C_{2}
$$

Similarly, $T 0 \cong \top$ so that this triple converts finite sums to $*$.

The dual statement is that if there is a right adjoint to the underlying functor from the category of counitary coassociative cocommutative coalgebras on $\mathscr{C}$ to $\mathscr{C}$, then the resultant cotriple will have the desired property. Hence we will consider the question of the existence of that adjoint.

The first case in which it exists is the case of a compact category. This is a *-autonomous category in which $A \multimap B \cong A^{\perp} \otimes B$. More precisely, the map

$$
A^{\perp} \otimes A \otimes B \stackrel{\text { eval } \otimes B}{\longrightarrow} \top \otimes B \cong B
$$


has a transpose $A \otimes B \longrightarrow A^{\perp} \multimap B$ and that map is required to be an isomorphism. In that case, the bifunctor $\otimes$ is cocontinuous and then the cofree coalgebra functor is given by letting, for an object $A, E_{n}(A)$ be the equalizer of the $n$ ! permutations of the $n$th tensor power $A^{\otimes n}$, for $n \geq 2$. Then

$$
! A=\perp \times A \times E_{2}(A) \times \cdots \times E_{n}(A) \times \cdots
$$

is the desired cotriple. The proof uses only that $\otimes$ commutes with countable products and is probably easier to see in the case. The dual construction of $E_{n} A$, called the $n$th symmetric power, is the coequalizer of the $n$ ! permutations.

Of the $*$-autonomous categories described in this paper, the only one that is compact is the category of sets and relations, in which not only is $A \multimap B=A^{\perp} \times B$, but also $A^{\perp}=A$ so that $A \multimap B=A \times B$. What is clearly necessary for the construction outlined above to work is that the category have finite products (or at least powers) and the equalizers used in the construction. The category is known to have arbitrary products (which are, in fact, the disjoint sums). It does not have all equalizers, but it has enough for our purposes:

5.1 Proposition. In the category of sets and relations, the simultaneous equalizer of any group of automorphisms exists. Thus that category gives a model of the full linear logic.

Proof. Given an object $A$ and a group $G$ of automorphisms, let $[a]$ denote the orbit of an element $a \in A$ under the action of $G$. Let $E$ be the set of orbits and $e: E$ $\longrightarrow A$ be the relation that assigns to an orbit the set of its elements. This is monic for if $f, g: B \longrightarrow E$ are relations such that $e \circ f=e \circ g$, then for any $b \in B$, we have $\bigcup f(b)=\bigcup g(b)$. If $f \neq g$, there would be some $a \in A$ such that $[a] \in f(b)$ while $[a] \notin g(b)$ or vice versa. Assuming this one holds, then $a \in \bigcup f(b)$, while $a \notin \bigcup g(b)$, because distinct orbits are disjoint.

Suppose now that $f: B \longrightarrow A$ is a relation that is equalized by all $\sigma \in G$. This clearly means that if $a \in f(b)$, then $[a] \subseteq f(b)$. But was can define $h: B \longrightarrow E$ by $h(b)=\{[a] \mid[a] \subseteq f(b)\}$. It is easy to see that $(e \circ g)(b)=\bigcup\{[a] \mid[a] \subseteq f(b)\}=f(b)$

The same considerations apply without essential change if we consider the category of relations in any topos that has at least countable limits. In fact, it is known that free algebras for finitary theories, such as that of commutative algebras, exist as soon as the topos has a natural numbers object. In this case, cofree coalgebras are the same as free algebras so that it is sufficient that the topos have a natural numbers object.

\section{$6 \quad$ Separated objects}

In this section, we will consider a modification of Chu's construction that in the case that we begin with a cartesian closed category, this modified category will have cofree coalgebras and be a model of the full linear logic. 
By "coalgebra" we will always mean a counitary, coassociative and cocommutative coalgebra. We begin with the observation that if $\mathscr{V}$ is an autonomous category and $\operatorname{Coalg}(\mathscr{V})$ the category of coalgebras, and if $U$ : Coalg $(\mathscr{V}) \longrightarrow \mathscr{V}$ is the underlying functor, then $U$ creates arbitrary colimits. It is thus plausible to suppose that cofree coalgebras exist, but proving it is another matter. On the other hand, if they should exist it is automatic that the resultant cotriple $\mathbf{G}=(G, \epsilon, \delta)$ on $\mathscr{V}$ will satisfy $G(V \times W) \cong G V \otimes G W$. and we will get a model of linear logic.

It turns out that if we apply Chu's construction to a cartesian closed category, then under reasonable hypotheses there is a certain full *-autonomous subcategory which has cofree coalgebras. We turn to this development. The first part is valid for any *-autonomous category $\mathscr{V}$ for which cofree coalgebras exist for $\mathscr{V}$.

Let us suppose that $\mathscr{V}$ is a symmetric monoidal closed category and that cofree coalgebras for the tensor already exist in $\mathscr{V}$. If $\mathscr{V}$ is cartesian closed, this condition is automatic. The reason is that every object in a cartesian closed has a unique coalgebra structure - the diagonal. In fact, the counit $V \rightarrow 1$ is the unique map and a map $V$ $\longrightarrow V \times V$ which is just counitary with respect to $V \longrightarrow 1$ is the diagonal. Since the diagonal is a coalgebra structure, unitary, coassociative and cocommutative, the claim follows. But in that case the identity cotriple is the cofree coalgebra cotriple.

We will also need a dualizing object we denote $\perp$. Before we discuss its properties, we have to say that we require a factorization system $(\mathscr{E}, \mathscr{M})$ which is compatible with the internal hom in the sense that if $f: V \longrightarrow V^{\prime}$ is in $\mathscr{E}$ and $g: W \longrightarrow W^{\prime}$ is in $\mathscr{M}$, then $f \multimap g: V^{\prime} \multimap W^{\prime} \longrightarrow V \multimap W$ is in $\mathscr{M}$. Then the additional property we need of $\perp$ is that it is an internal cogenerator with respect to this factorization system, which means that the natural map $V \longrightarrow V^{\perp \perp}$ belongs to $\mathscr{M}$. We will suppose that the arrows in $\mathscr{E}$ are epimorphisms.

We will use the notation $f: V \rightarrow V^{\prime}$ when $f \in \mathscr{E}$ and $g: W \longmapsto W^{\prime}$ when $g \in \mathscr{M}$.

\subsection{Proposition. The following hold:}

1. If $f: V^{\prime} \rightarrow V$ then for any $W, f \otimes W: V^{\prime} \otimes W \rightarrow V \otimes W$.

2. If $f: V^{\prime} \longrightarrow V$ and $g: W \succ W^{\prime}$, then

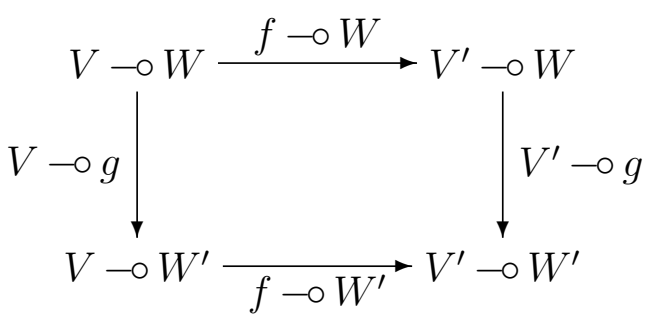

is a pullback. 
3. If $f: V^{\prime} \rightarrow V$ and $g: W^{\prime} \rightarrow W$, then

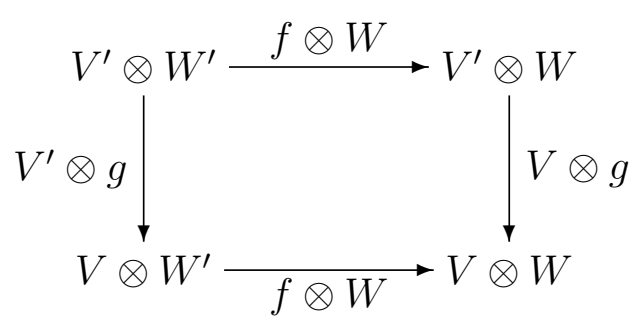

is a pushout.

Proof. The key to this is the observation that the diagonal fill-in of a factorization system is equivalent to the hypothesis that whenever $f: V^{\prime} \rightarrow V$ and $g: W \longmapsto W^{\prime}$, then

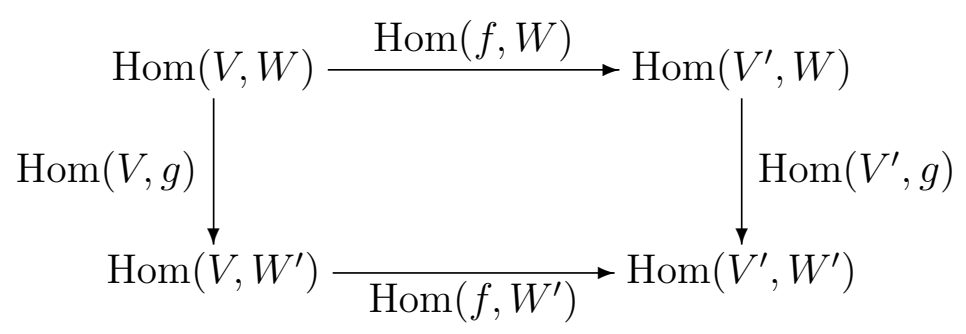

is a pullback. This is, in fact the external version of item 2. We now turn to the individual parts of the proof.

1. We have to show that for any $U \longmapsto U^{\prime}$, the square

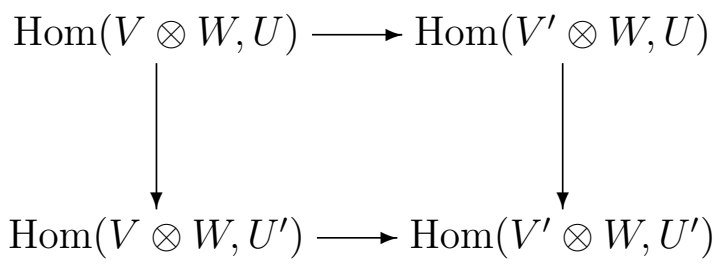

is a pullback. By adjointness, this is equivalent to

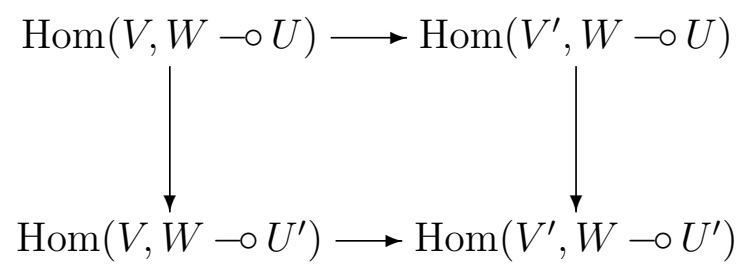

which is a pullback because $V \rightarrow V^{\prime}$ and $W \multimap U \succ W \multimap U^{\prime}$.

2. We have to show that for any $U$, the square

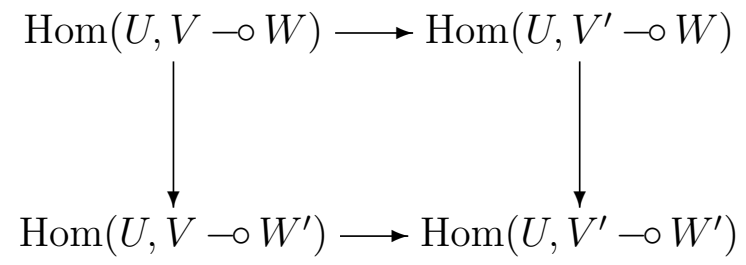


is a pullback. By adjointness, this is equivalent to the square

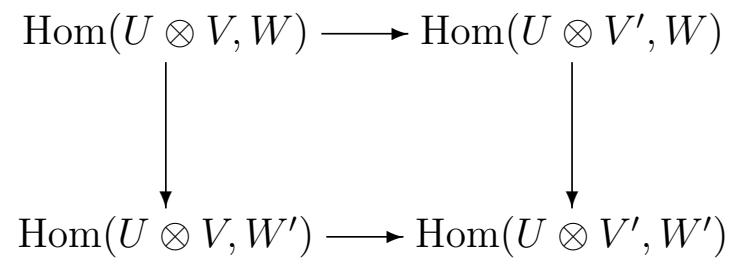

which follows from the first part.

3. We must show that for any $U \longrightarrow U^{\prime}$, the square

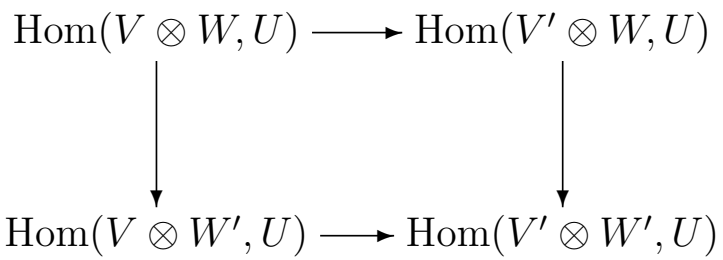

is a pullback. By adjointness, this is equivalent to

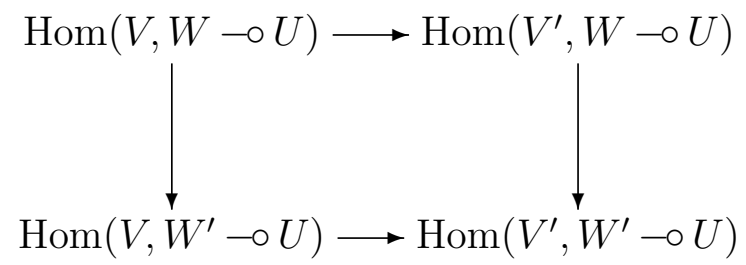

which follows from $V^{\prime} \rightarrow V$ and $W^{\prime} \multimap U \succ W \multimap U$.

Let $\mathscr{A}=\mathscr{V}_{\perp}$ be the Chu category that results from this choice of dualizing object. We will most often denote the object $\left(V, V^{\prime}, v\right)$ by simply $\left(V, V^{\prime}\right)$, suppressing the structure map. The arrow $v$ induces by transposition arrows $V \longrightarrow V^{\prime \perp}$ and $V^{\prime} \longrightarrow V^{\perp}$. Say the object $\left(V, V^{\prime}\right)$ is left separated if the first of these arrows is a monomorphism and right separated if the second one is and separated if it satisfies both conditions. We denote the full subcategories of objects satisfying these three conditions by $\mathscr{A}_{l}, \mathscr{A}_{r}$ and $\mathscr{A}_{s}$, respectively.

6.2 Proposition. There are functors $l: \mathscr{A} \longrightarrow \mathscr{A}$ and $r: \mathscr{A} \longrightarrow \mathscr{A}$ that reflect and coreflect, respectively, $\mathscr{A}$ to $\mathscr{A}_{l}$ and $\mathscr{A}$ to $\mathscr{A}_{r}$. Moreover $r$ and l commute with each other.

Proof. Let $A=\left(V, V^{\prime}\right)$ be an object. Factor $V \longrightarrow V^{\prime \perp}$ as $V \rightarrow \widetilde{V} \longmapsto V^{\prime \perp}$. Let $l A=\left(\widetilde{V}, V^{\prime}\right)$. We have the arrow $\left(V, V^{\prime}\right) \longrightarrow\left(\widetilde{V}, V^{\prime}\right)$. If $\left(f, f^{\prime}\right):\left(V, V^{\prime}\right) \longrightarrow\left(W, W^{\prime}\right)$ is a 
morphism in $\mathscr{A}$ and $\left(W, W^{\prime}\right)$ is in $\mathscr{A}_{l}$, then in the commutative diagram

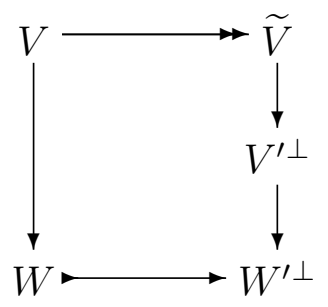

the diagonal fill-in gives the map $\widetilde{V} \rightarrow W$ required to factor $f$ through $l A$. Since clearly $l A$ is in $\mathscr{A}_{l}$, the adjointness follows. A similar argument works for $\mathscr{A}_{r}$. To see that the functors commute, we observe that from $V \rightarrow \widetilde{V}$ follows $\widetilde{V}^{\perp} \longmapsto V^{\perp}$ and then the images of $V^{\prime} \longrightarrow \widetilde{V}^{\perp}$ and $V^{\prime} \longrightarrow V^{\perp}$ are the same, so you get the same result whether you apply $l$ before or after you apply $r$.

It follows from the fact that $r$ commutes with $l$ that $\mathscr{A}_{s}$ is a reflective subcategory of $\mathscr{A}_{r}$ (with reflector $l$ ) and a coreflective subcategory of $\mathscr{A}_{l}$ (with coreflector $r$ ). From this it follows that if $\mathscr{A}$ is complete (=cocomplete), then so is $\mathscr{A}_{s}$.

We call attention to the very important,

6.3 Theorem. [Recognition theorem] If $\mathscr{B}$ is a full reflexive subcategory of $\mathscr{C}$ with reflector $F$, then a necessary and sufficient condition that the object $B$ be isomorphic to an object of $\mathscr{B}$ is that for all objects $C$ of $\mathscr{C}$, the adjunction morphism $C \longrightarrow F C$ induces an isomorphism

$$
\operatorname{Hom}(F C, B) \stackrel{\cong}{\longrightarrow} \operatorname{Hom}(C, B)
$$

The proof is simple and is left to the reader.

It thus follows from the dual form that if $A$ is an object of $\mathscr{A}_{r}$ and $B$ an object of $\mathscr{A}$, then $\operatorname{Hom}(A, r B) \rightarrow \operatorname{Hom}(A, B)$ is an isomorphism. The $\mathscr{V}$-version is also true:

6.4 Proposition. If $A$ is an object of $\mathscr{A}_{r}$ and $B$ an object of $\mathscr{A}$, then $\mathscr{V}(A, r B)$ $\longrightarrow \mathscr{V}(A, B)$ is an isomorphism.

Proof. Let $A=\left(V, V^{\prime}\right), B=\left(W, W^{\prime}\right)$ and $r B=(W, \widetilde{W})$. We have $W^{\prime} \rightarrow \widetilde{W}$ and $V^{\prime} \longmapsto V^{\perp}$ so that the right hand square of

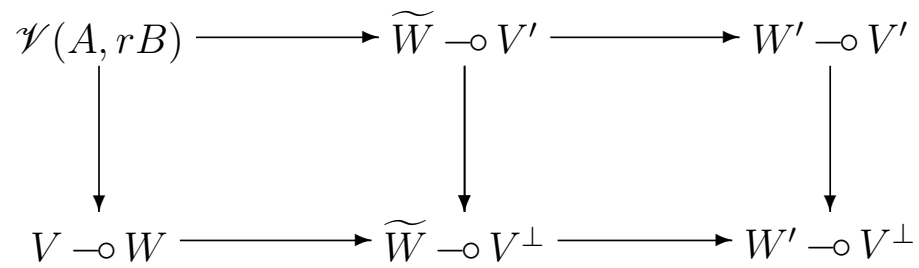

is a pullback. The left hand square is also a pullback by the definition of $\mathscr{V}(A, r B)$ and hence the outer rectangle is. But that is the definition of $\mathscr{V}(A, B)$. 
6.5 Corollary. If $A$ is in $\mathscr{A}_{r}$ and $B$ is in $\mathscr{A}$, then $r(A \multimap r B) \longrightarrow r(A \multimap B)$, induced by $r B \longrightarrow B$, is an isomorphism.

Proof. If $A=\left(V, V^{\prime}\right)$ and $B=\left(W, W^{\prime}\right)$, then $A \multimap B=\left(\mathscr{V}(A, B), V \otimes W^{\prime}\right)$. We have that $r(A \multimap B)=(\mathscr{V}(A, r B), X)$ where $X$ is the image of $V \otimes W^{\prime} \longrightarrow \mathscr{V}(A, B)^{\perp}$. In a similar way, if $r B=(W, \widetilde{W})$, then $r(A \multimap r B)=(\mathscr{V}(A, r B), Y)$, where $Y$ is the image of $V \otimes \widetilde{W} \longrightarrow \mathscr{V}(A, r B)^{\perp}$. We have from the previous proposition that $\mathscr{V}(A, r B) \cong \mathscr{V}(A, B)$. Using this and the fact that $\otimes$ preserves $\mathscr{E}$ (item 1 of 6.1 ), we have the following square:

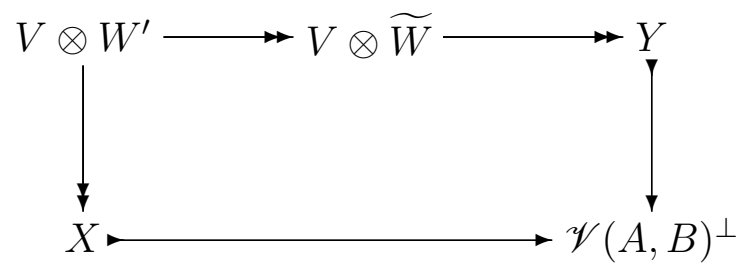

whose diagonal fill-ins in each direction give the required isomorphism.

6.6 Corollary. If $A$ and $B$ are in $\mathscr{A}_{r}$, then so is $A \otimes B$. If $A$ is in $\mathscr{A}_{r}$ and $B$ in $\mathscr{A}_{l}$, then $A \multimap B$ is in $\mathscr{A}_{l}$

Proof. We have that for all $C$ in $\mathscr{A}$,

$$
\begin{aligned}
\operatorname{Hom}(A \otimes B, C) & \cong \operatorname{Hom}(A, B \multimap C) \cong \operatorname{Hom}(A, r(B \multimap C)) \\
& \cong \operatorname{Hom}(A, r(B \multimap r C)) \cong \operatorname{Hom}(A, B \multimap r C) \cong \operatorname{Hom}(A \otimes B, r C)
\end{aligned}
$$

and the conclusion follows from the recognition theorem. For the second claim, the hypotheses imply that $A$ and $B^{\perp}$ are in $\mathscr{A}_{r}$ whence so is $A \otimes B^{\perp}$ so that $A \multimap B \cong$ $\left(A \otimes B^{\perp}\right)^{\perp}$ is in $\mathscr{A}_{l}$.

6.7 Corollary. The category $\mathscr{A}_{s}$ is a *-autonomous category with the same tensor unit and dualizing object and with tensor product $l(A \otimes B)$ and internal hom $r(A \multimap B)$.

Proof. The tensor unit $(T, \perp)$ belongs to $\mathscr{A}_{s}$ because our hypothesis that $\perp$ is an internal generator implies that $\top \longrightarrow \perp^{\perp}$ is monic, while the other arrow that has to be monic is the identity of $\perp$. The previous corollary implies that $l(A \otimes B)$ and $r(A \multimap B)$ actually belong to $\mathscr{A}_{s}$. For objects $A, B$ and $C$ of $\mathscr{A}_{s}$, we have

$$
\operatorname{Hom}(l(A \otimes B), C) \cong \operatorname{Hom}(A \otimes B, C) \cong \operatorname{Hom}(A, B \multimap C) \cong \operatorname{Hom}(A, r(B \multimap C))
$$

Since $(\top, \perp) \otimes A \cong(\top, \perp) \multimap A \cong A$ and $A \multimap \perp \cong A^{\perp}$ already belong to $\mathscr{A}_{s}$, those constructions are the same as they are in $\mathscr{A}$ and so $\mathscr{A}_{s}$ has the same tensor unit and dualizing object as $\mathscr{A}$. 


\section{$7 \quad \mathscr{V}$ as a subcategory of $\mathscr{A}$}

There is a functor $I: \mathscr{V} \longrightarrow \mathscr{A}$ that takes $V$ to the object $\left(V, V^{\perp}\right)$. It is obvious that $I V$ is always an object of $\mathscr{A}_{r}$ and since we have made the hypothesis that the map $V$ $\longrightarrow V^{\perp \perp}$ is monic, it is actually an object of $\mathscr{A}_{s}$. We claim that $I$ is full and faithful. In fact, an arrow $\left(V, V^{\perp}\right) \longrightarrow\left(W, W^{\perp}\right)$ consists of a map $f: V \longrightarrow W$ and a map $f^{\prime}: W^{\perp}$ $\rightarrow V^{\perp}$ such that

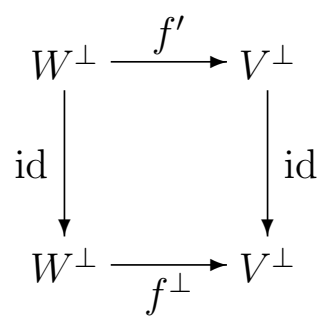

commutes, which implies that $f^{\prime}=f^{\perp}$. It is easy to see that $I$ actually factors through $\mathscr{A}_{s}$ and hence through $\mathscr{A}_{r}$ and $\mathscr{A}_{l}$. Let $I_{r}, I_{l}$ and $I_{s}$ denote the functors to those categories whose values are the same as those of $I$.

7.1 Proposition. The functors $I, I_{r}, I_{l}$ and $I_{s}$ preserve the tensor.

Proof. We have $I V \otimes I W=\left(V \otimes W, \mathscr{V}\left(I V,(I W)^{\perp}\right)\right)$. The definition of $\mathscr{V}\left(I V,(I W)^{\perp}\right)$ is that the square

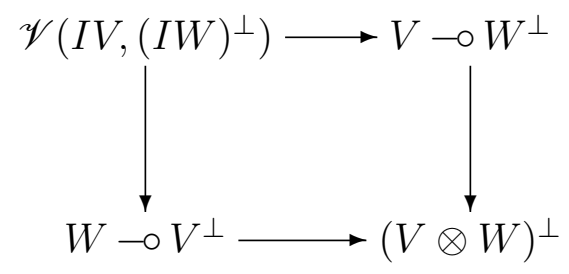

is a pullback. But the lower and right hand arrows are isomorphisms in any symmetric closed monoidal category, whatever the dualizing object (it is just the usual hom/tensor adjointness, plus symmetry) and so we have that $\left.\mathscr{V}\left(I V,(I W)^{\perp}\right)=(V \otimes W)^{\perp}\right)$. This takes care of $I$ and since $I(V \otimes W) \cong I(V) \otimes I(W)$, the latter is the tensor product in all four categories, which means there is no need to reflect.

7.2 Proposition. The forgetful functor $\check{I}: \mathscr{A} \longrightarrow \mathscr{V},\left(V, V^{\prime}\right) \mapsto V$, is right adjoint to $I$, which is thereby the inclusion of a coreflective subcategory.

Proof. If $V$ is an object of $\mathscr{V}$ and $\left(W, W^{\prime}\right)$ an object of $\mathscr{A}$, then for any $f: V \rightarrow W$ there is clearly a unique arrow $f^{\prime}: W^{\prime} \longrightarrow V^{\perp}$ such that

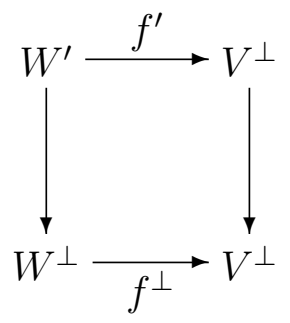


commutes.

7.3 Corollary. The forgetful functors $\check{I}_{r}: \mathscr{A}_{r} \longrightarrow \mathscr{V}, \check{I}_{l}: \mathscr{A}_{l} \longrightarrow \mathscr{V}$ and $\check{I}_{s}: \mathscr{A}_{s} \longrightarrow \mathscr{V}$ are right adjoint to $I_{r}, I_{l}$ and $I_{s}$, respectively.

Let $\operatorname{Coalg}(\mathscr{V})$ and $\operatorname{Coalg}(\mathscr{A})$ denote the category of cocommutative counitary coalgebras over $\mathscr{V}$ and $\mathscr{A}$ respectively. If $c: V \longrightarrow V \otimes V$ and $p: V \longrightarrow \top$ are the structure maps of a coalgebra then there is a coalgebra structure on $I V=\left(V, V^{\perp}\right)$. This is immediate because $I$ is full and faithful and preserves the tensor and unit object. This gives a functor $J$ : $\operatorname{Coalg}(\mathscr{V}) \longrightarrow \operatorname{Coalg}(\mathscr{A})$.

Since $A \otimes A=\left(V \otimes V, \mathscr{V}\left(A, A^{\perp}\right)\right)$, if $A=\left(V, V^{\prime}\right)$ has a coalgebra structure $\left(c, c^{\prime}\right): A$ $\longrightarrow A \otimes A$ and $\left(p, p^{\prime}\right): A \longrightarrow(\top, \perp)$, then $c: V \longrightarrow V \otimes V$ and $p: V \longrightarrow \top$ give a coalgebra structure on $V$. This defines a functor $\check{J}$ : $\operatorname{Coalg}(\mathscr{A}) \longrightarrow \operatorname{Coalg}(\mathscr{V})$. The forgotten part of the coalgebra structure, $c^{\prime}$ and $p^{\prime}$, turn out to be equivalent to $\left(c, c^{\prime}\right): I V \longrightarrow\left(V, V^{\prime}\right)$ being morphisms of coalgebras. The same argument works for $\mathscr{A}_{r}$, because it is closed under tensor product. full subcategories, preserves the tensor. We denote that functor by $\check{J}_{r}: \operatorname{Coalg}\left(\mathscr{A}_{r}\right) \rightarrow \operatorname{Coalg}(\mathscr{V})$.

7.4 Proposition. The functors $J$ and $J_{r}$ are full and faithful and are left adjoint to $\check{J}$ and $\check{J}_{r}$, respectively.

Proof. The first claim is the routine verification that a coalgebra homomorphism from $\left(V, V^{\perp}\right) \longrightarrow\left(W, W^{\perp}\right)$ is induced by a unique coalgebra homomorphism $V \rightarrow W$. For the adjointness, suppose that $V$, together with $c: V \longrightarrow V \otimes V$ and $p: V \longrightarrow \top$ is a coalgebra in $\mathscr{V}$ and $A=\left(W, W^{\prime}\right)$ with structure maps $\left(d, d^{\prime}\right)$ and $\left(q, q^{\prime}\right)$ is a coalgebra structure in $\mathscr{A}$ or in $\mathscr{A}_{r}$. Suppose that $f: V \rightarrow W$ is a coalgebra morphism from $(V, c, p)$ to $(W, d, q)$. Consider first the case of $\mathscr{A}$. Define the arrow $f^{\prime}: W^{\prime} \longrightarrow V^{\perp}$ as the composite $W^{\prime} \longrightarrow W^{\perp} \longrightarrow V^{\perp}$, the second arrow being $f^{\perp}$. The diagram that must be shown to commute is the outer rectangle in the diagram

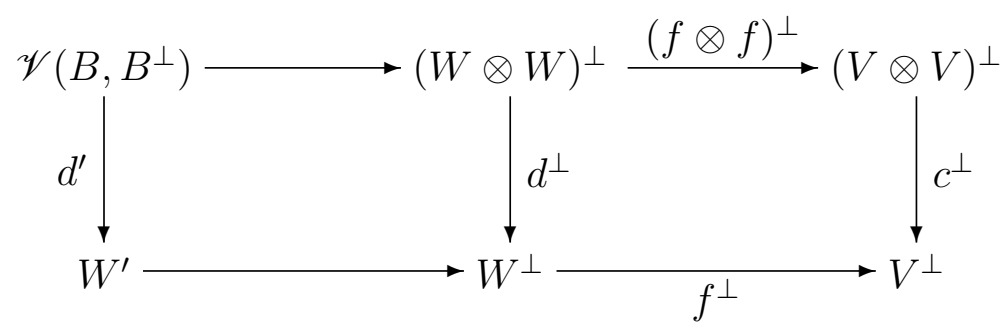

The left hand square commutes in order that $\left(d, d^{\prime}\right)$ be an arrow and the right hand square is just the dual of the commutative square that says that $f$ is a coalgebra morphism in Coalg $(\mathscr{V})$. The argument involving $\mathscr{A}_{r}$ is similar, with $\mathscr{V}\left(B, B^{\perp}\right)$ replaced by its image in $(W \otimes W)^{\perp}$.

7.5 Proposition. The adjunction morphisms $I_{r} \check{I}_{r} \longrightarrow$ id and $J_{r} \check{J}_{r} \longrightarrow$ id are epimorphisms. 
Proof. If $\left(V, V^{\prime}\right)$ is an object of $\mathscr{A}_{r}$, then $V^{\prime} \longrightarrow V^{\perp}$ is monic which implies that the arrow $\left(V, V^{\perp}\right) \longrightarrow\left(V, V^{\prime}\right)$ is epic.

The situation we have then can be described by the following diagram, which commutes read both ways, in which the lower arrow in each line is left adjoint to the upper one and the vertical arrows are the forgetful functors.

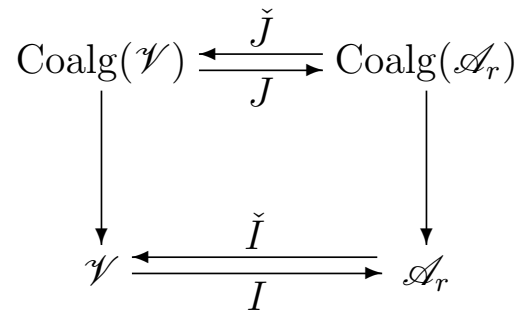

\section{An adjoint interrupt}

At this point it is worth the effort to abstract away from the particular situation we are in and prove a theorem on the existence of adjoints which will apply to this situation.

8.1 Theorem. Suppose we have a diagram of categories and functors of the following form:

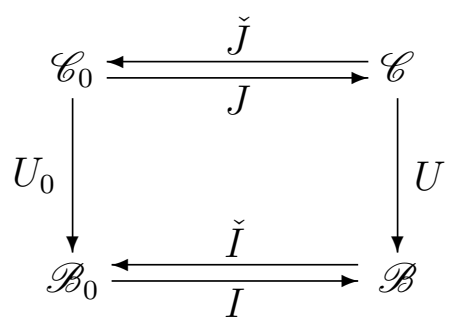

Suppose that $\mathscr{E}$ is a class of epimorphisms in $\mathscr{C}$ and that

1. $\mathscr{E}$ is stable under pushouts;

2. I is left adjoint to $\check{I}$ with adjunction morphism $\epsilon_{I}: I \check{I} \longrightarrow \mathrm{id}$;

3. $J$ is left adjoint to $\check{J}$ and the adjunction morphism $\epsilon_{J}: J \breve{J} \longrightarrow$ id is in $\mathscr{E}$;

4. $U \circ J=I \circ U_{0}, U_{0} \circ \check{J}=\check{I} \circ U$ and $\epsilon_{I} U=U \epsilon_{J}$;

5. $\mathscr{C}$ has and $U$ preserves colimits;

6. $\mathscr{C}$ is $\mathscr{E}$-well-co-powered;

7. $U_{0}$ has a right adjoint. 


\section{Then $U$ has a right adjoint.}

Of course, the equalities in the fourth item could be replaced by isomorphisms, in which case the last clause of that item would have to modified.

Proof. It is sufficient to show that there is a solution set for the adjoint. Let $R_{0}$ denote the right adjoint of $U_{0}$ and let $\epsilon_{0}: U_{0} R_{0} \longrightarrow$ id denote the back adjunction. What we will show is that for an object $B$ of $\mathscr{B}$ the set of maps whose domain is an $\mathscr{E}$-quotient of the object $J R_{0} \check{I} B$ constitutes a solution set. Suppose $f: U C \longrightarrow B$ is a morphism in $\mathscr{B}$. Then $\check{I} f: \breve{I} U C=U_{0} \breve{J} C \longrightarrow \breve{I} B$ is an arrow of $\mathscr{B}_{0}$ and so there is a unique arrow $f^{\sharp}: \breve{J} C \longrightarrow R_{0} \check{I} B$ such that $\check{I} f=\epsilon_{0} \check{I} B \circ U_{0} f^{\sharp}$. Let $C^{\prime}$ be defined by forming the pushout

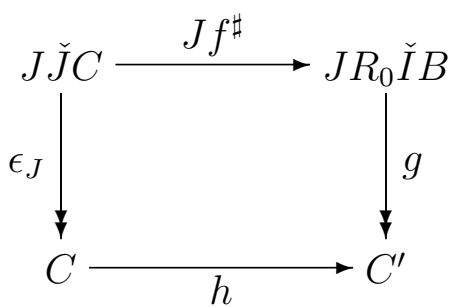

Since $U$ preserves colimits, the square

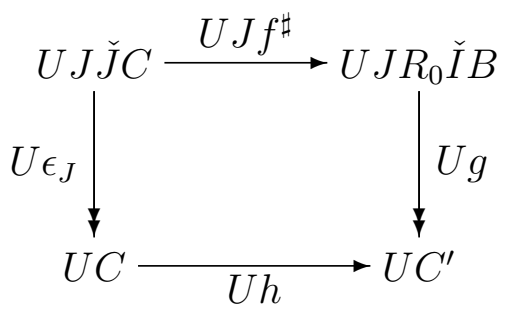

is also a pushout. We calculate, using that $U J R_{0} \check{B}=I U_{0} R_{0} \check{I} B$ and $U J \breve{J} C=I \check{I} U_{0} C$,

$$
\begin{aligned}
\epsilon_{I} B \circ I \epsilon_{0} \check{I} B \circ U J f^{\sharp} & =\epsilon_{I} B \circ I \epsilon_{0} \check{I} B \circ I U_{0} f^{\sharp}=\epsilon_{I} B \circ I\left(\epsilon_{0} \check{I} \circ U_{0} f^{\sharp}\right) \\
& =\epsilon_{I} B \circ I \check{I} f=f \circ \epsilon_{I} U C=f \circ U \epsilon_{J}
\end{aligned}
$$

which means there is a unique $k: U C^{\prime} \longrightarrow B$ such that $k \circ U h=f$ and $k \circ U g=$ $\epsilon_{I} B \circ I \epsilon_{0} \check{I} B$. The first of these identities shows that $f$ factors through the map underlying an $\mathscr{E}$-quotient of $U J R_{0} \check{I} B$ thus those maps form a solution set.

\section{Cofree coalgebras}

We now apply this theorem, with $\mathscr{E}$ the class of all arrows of the form (id, $\left.f^{\prime}\right):\left(V, V^{\prime}\right)$ $\longrightarrow\left(W, W^{\prime}\right)$ for which $f^{\prime}$ is a monomorphism in $\mathscr{V}$. To see this is closed under pushouts in $\operatorname{Coalg}\left(\mathscr{A}_{r}\right)$, we observe that both the forgetful functor and the inclusion of $\mathscr{A}_{r}$ into $\mathscr{A}$ preserve colimits. This class of arrows in $\mathscr{A}$ is closed under pushouts since monics are closed under pullbacks. 
9.1 Theorem. Let $\mathscr{V}$ be a well-co-powered complete and cocomplete autonomous category. Let $\perp$ be an internal cogenerator in $\mathscr{V}$. Then the category $\mathscr{A}_{r}$ of right separated objects in the Chu category $\mathscr{A}=\mathscr{V}_{\perp}$ has cofree coalgebras.

This is interesting, but still not an example of full linear logic because $\mathscr{A}_{r}$ is not *-autonomous, since it is not self dual. $\mathscr{A}_{s}$ is self dual, but we haven't shown that it has cofree coalgebras, only that it is a full subcategory of a category that does. Perhaps this can be used to show that $\mathscr{A}_{s}$ has cofree coalgebras in general, but we can do this only in one special case: that of a cartesian closed category.

What is special about a cartesian closed category is that there is only the terminal map $p: V \longrightarrow \top=1$ and if $c: V \longrightarrow V \times V$ is a counitary comultiplication, then id $\times p: V \times V \rightarrow V$ is just the first projection and $p \times$ id is the second so that the equations (id $\times p) \circ c=$ id and $(p \times$ id $) \circ c=$ id are satisfied uniquely by the diagonal map. Thus if $\mathscr{V}$ is cartesian closed and $A=\left(V, V^{\prime}\right)$ is a coalgebra in $\mathscr{A}_{l}$, then there are morphisms $\left(V, V^{\prime}\right) \longrightarrow l\left(V \times V, \mathscr{V}\left(A, A^{\perp}\right)\right)$ and $\left(p, p^{\prime}\right):\left(V, V^{\prime}\right) \longrightarrow(1, \perp)$ satisfying the same equations (and others: associativity and commutativity). We have $l(V \times$ $\left.V, \mathscr{V}\left(A, A^{\perp}\right)\right)=\left(W, \mathscr{V}\left(A, A^{\perp}\right)\right)$ with $V \times V \rightarrow W$. But the equations imply that $V \times V$ $\longrightarrow W$ is split monic, split by $\langle$ id $\otimes p, p \otimes$ id $\rangle$, where $\otimes$ is the tensor product in $\mathscr{A}_{l}$. Since we assume the arrows in $\mathscr{E}$ are epic, this implies this one is an isomorphism and

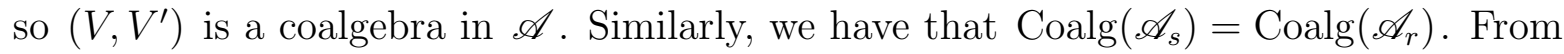
this it is immediate that the underlying functor Coalg $\left(\mathscr{A}_{s}\right) \longrightarrow \mathscr{A}_{s}$ has a right adjoint, just the inclusion into $\mathscr{A}_{r}$ followed by that adjoint. Thus we have:

9.2 Theorem. Let $\mathscr{V}$ be a complete and cocomplete cartesian closed category and let $\perp$ be an internal cogenerator. Then the category of separated objects for the Chu category $\mathscr{V}_{\perp}$ is a model of full linear logic.

One obvious source of examples of this is to take $\mathscr{V}$ to be any Grothendieck topos and $\perp$ to be the subobject classifier, which is internally an injective cogenerator.

\section{Example}

Let $\mathscr{V}$ be the category of sets and $\perp=2$, the two element set. Then $\mathscr{A}_{s}$ has as objects $\left(X, X^{\prime}\right)$ where $X$ is a set and, up to isomorphism, $X^{\prime}$ is a set of subsets of $X$ that separates points in the sense that for any two elements of $X$, there is some set in $X^{\prime}$ that contains one of the elements and not the other. An example of such a pair is gotten from a $\mathrm{T}_{0}$ topology on $X$ that takes for $X^{\prime}$ the set of open sets. It turns out that $\left(X, X^{\prime}\right) \otimes\left(Y, Y^{\prime}\right)=\left(Z, Z^{\prime}\right)$ where $Z^{\prime}$ is the set of all relations $R \subseteq X \times Y$ such that for each $x \in X$, the set of elements of $Y$ that are $R$-related to $x$ is in $Y^{\prime}$ and conversely, for each $y \in Y$ that are $R$-related to $y$ is in $X^{\prime}$. The set $Z$ is the separated quotient of $X \times Y$. That is, two elements of $X \times Y$ are identified if every such relation that contains one of them contains the other. If $A=\left(X, X^{\prime}\right)$ is to have the structure of 
a coalgebra, then it must be that the tensor product in $\mathscr{A}$ is already separated, so that we need a map $\left(c, c^{\prime}\right):\left(X, X^{\prime}\right) \longrightarrow\left(X \times X\right.$, $\left.\operatorname{Set}\left(A, A^{\perp}\right)\right)$ as well as a map $\left(p, p^{\prime}\right):\left(X, X^{\prime}\right)$ $\longrightarrow(1, \perp)$. There is no choice for $c$ and $p$ which must be the diagonal and terminal maps, respectively. It turns out that there is also no choice for $p^{\prime}$ which must have the values $p^{\prime}(0)=\emptyset$ and $p^{\prime}(1)=X$, thus forcing those two subsets to belong to $X^{\prime}$. As for $c^{\prime}$, there is also no choice. If $f: X \longrightarrow X^{\prime}$ is a function, then we can think of $f: X \longrightarrow X \multimap 2$ and it has a graph $G(f)$. Say that $f$ is admissible if both $G(f)$ and $G(f)^{\text {op }}$ (the opposite graph) are the graphs of functions $X \rightarrow X^{\prime}$. The set $\operatorname{Set}\left(A, A^{\perp}\right)$ can be identified as the set of admissible maps. Then the condition on the existence of $c^{\prime}$ is that for any admissible $f,\{x \mid(x, x) \in G(f)\}$ belong to $X^{\prime}$ and then $c^{\prime}(f)$ is just that set.

Just to show that there are examples (other, of course, than $\left(X, X^{\perp}\right)$ which always works), here is an example of a separated coalgebra in the Chu category of sets. We take $X=2$ and $X^{\prime}=\{\emptyset,\{0\}, X\} \subseteq X \multimap 2$ and $A=\left(X, X^{\prime}\right)$. The coalgebra structure is a pair $\left(c, c^{\prime}\right)$ where $C: X \longrightarrow X \times X$ is the diagonal map and $c^{\prime}: \mathscr{V}\left(A, A^{\perp}\right) \longrightarrow X^{\prime}$ is as described below. An element of $\mathscr{V}\left(A, A^{\perp}\right)$ is a pair $(f, g)$ where $f, g: X \longrightarrow X^{\prime}$ are opposites of each other. That is, if we follow $f$ and $g$ by the arrows into $X \multimap 2$, we get two binary relations on $X$ and these should be the opposite. In the table below, we tabulate the possible maps $f: X \longrightarrow X^{\prime}$, the opposite maps $g: X \longrightarrow X \multimap 2$ and when the latter factors through $X^{\prime}$ (and thus gives a map of $A$ to $A^{\perp}$ ), the value $c^{\prime}(f, g)$. A dash in the last column means that that row does not correspond to a map from $A$ to $A^{\perp}$.

\begin{tabular}{cc|cc|c}
$f(0)$ & $f(1)$ & $g(0)$ & $g(1)$ & $c^{\prime}(f, g)$ \\
\hline$\emptyset$ & $\emptyset$ & $\emptyset$ & $\emptyset$ & $\emptyset$ \\
$\emptyset$ & $\{0\}$ & $\{1\}$ & $\emptyset$ & - \\
$\emptyset$ & $X$ & $\{1\}$ & $\{1\}$ & - \\
$\{0\}$ & $\emptyset$ & $\{0\}$ & $\emptyset$ & $\{0\}$ \\
$\{0\}$ & $\{0\}$ & $X$ & $\emptyset$ & $\{0\}$ \\
$\{0\}$ & $X$ & $X$ & $\{1\}$ & - \\
$X$ & $\emptyset$ & $\{0\}$ & $\{0\}$ & $\{0\}$ \\
$X$ & $\{0\}$ & $X$ & $\{0\}$ & $\{0\}$ \\
$X$ & $X$ & $X$ & $X$ & $X$
\end{tabular}

The commutativity of this (which is automatic, given that of $c$ ) is shown in the fact that nothing changes when $f$ and $g$ are interchanged. The fact that it is counitary is equivalent to the fact that $\emptyset$ and $X$ are in $X^{\prime}$.

\section{Why simple recursion doesn't work}

One approach to the existence of cofree coalgebras that looks attractive but doesn't work is to try to solve the recursive equation $\Phi(A)=\top \times(A \otimes \Phi(A))$. I leave aside the 
fact that the solution to this equation won't be cocommutative, since that may suffice for the requirements of linear logic. The real problem is that the elementary recursion implicit in this equation simply doesn't define anything.

It certainly looks like it works. If you write

$$
\Phi(A)=\top \times(A \otimes(\top \times(A \otimes(\top \times(A \otimes \cdots)))))
$$

the equation $\Phi(A)=\top \times(A \otimes \Phi(A)$ seems to be satisfied by a direct substitution (try it!). The problem comes when you try to give a serious interpretation of the $\cdots$.

Here is the most obvious interpretation of the limiting process. Let $\Phi_{0}$ be the constant functor $\Phi_{0}(A)=\top$ and let $\Phi_{1}(A)=\top \times\left(A \otimes \Phi_{0}(A)\right)$. Let $\phi_{0}: \Phi_{1} \longrightarrow \Phi_{0}$ be the natural transformation induced by the projection on the first factor. Having defined functors $\Phi_{0}, \Phi_{1}, \ldots, \Phi_{n}$ and natural transformations $\phi_{i-1}: \Phi_{i} \longrightarrow \Phi_{i-1}$ for $0 \leq i<n$, so that $\Phi_{i}(A)=\top \times\left(A \otimes \Phi_{i-1}\right.$ for $1 \leq i<n$, let $\Phi_{n+1}(A)=\top \times\left(A \otimes \Phi_{n}(A)\right)$ and $\phi_{n}=\mathrm{id}_{\top} \times\left(\mathrm{id}_{A} \otimes \phi_{n-1}\right)$. Let $\Phi(A)$ be the inverse limit of the sequence.

Now if you try to show that $\Phi(A) \cong \top \times(A \otimes \Phi(A))$, you will get the sequence

$$
A \otimes \Phi(A) \longrightarrow \cdots A \otimes \Phi_{n}(A) \longrightarrow A \otimes \Phi_{n-1}(A) \longrightarrow \cdots \longrightarrow A \otimes \Phi_{0}(A)
$$

If this were a limit (that is if $A \otimes$ preserved the limit of the sequence that defines $\Phi$ ), then could conclude that

$$
\top \times A \otimes \Phi(A) \longrightarrow \cdots \top \times A \otimes \Phi_{n}(A) \longrightarrow \top \times A \otimes \Phi_{n-1}(A) \longrightarrow \cdots \longrightarrow \top \times A \otimes \Phi_{0}(A)
$$

is also a limit. But it is the same as the sequence

$$
\cdots \longrightarrow \Phi_{n+1}(A) \longrightarrow \Phi_{n}(A) \longrightarrow \cdots \longrightarrow \Phi_{1}(A)
$$

whose limit is evidently $\Phi(A)$. The problem is that in most cases, there is no reason for $\otimes$ to commute with any inverse limit and, failing that, the argument breaks down. If the category were colocally presentable (see [Makkai \& Paré, 1990]), this could be done by taking the limit out to some sufficiently large ordinal, but a self dual category cannot be colocally presentable unless it is a complete lattice.

\section{Related works}

The category constructed by $\mathrm{Chu}$ is different from the categories constructed by de Paiva in either [de Paiva, 1989a] or [de Paiva, 1989b]. If you take $\mathscr{V}=$ Set and $\perp=2$, you get a category whose objects are the same as the categories in those papers, but the arrows are a bit different in each case. In fact, de Paiva's constructions work without a dualizing object and it is crucial for Chu's. On the other hand, she has to restrict to decidable subobjects to get the duality, while here it is automatic. 
On the other hand, the special case $\mathscr{V}=$ Set and $\perp=2$ was rediscovered by Lafont (he gave the objects the odd name of "games") in [Lafont, 1988]. He claims that the category $\mathscr{V}_{\perp}$ has cofree coalgebras in that special case, but gives only the barest sketch of a proof.

\section{References}

M. Barr, *-Autonomous categories. Lecture Notes in Mathematics 752, 1979, Springer-Verlag, Berlin, Heidelberg, New York.

P.-H. Chu, Constructing *-autonomous categories. Appendix to [Barr, 1979].

C. B. Jay, Languages for monoidal categories. J. Pure Applied Algebra, 59 (1989), $61-85$.

S. Eilenberg and G. M. Kelly, Closed categories. Proc. Conf. Categorical Algebra (La Jolla, 1965), Springer (1966), 421-562.

Y. Lafont, From linear algebra to logic. Preprint, 1988.

M. Makkai and R. Paré, Accessible Categories. Contemporary Mathematics, 104, Amer. Math. Soc. 1990.

V. C. V. de Paiva, The Dialecta categories. In J. Gray and A. Scedrov eds., Contemporary Mathematics, 92, (1989a), 47-62.

V. C. V. de Paiva, A Dialecta-like model of linear logic. In D. H. Pitt et al., eds. Category Theory and Computer Science, Lecture Notes in Computer Science 389, (1989b), 341-356.

R. A. G. Seely, Linear logic, *-autonomous categories and cofree coalgebras. In J. Gray and A. Scedrov, eds., Categories in Computer Science and Logic, Contemporary Mathematics, 92, Amer. Math. Soc. (1989), 371-382. 